\section{Goals change crowd air chemistry}

During live public screenings of the 2016 UEFA European Championships, the emission rates of particular chemicals in the audience's breath vary sharply - apparently in response to events on the football pitch.

Football matches induce fans to roar in jubilation, hold their breath in suspense and sigh with disappointment. On 26 June, we tracked reactions from a cinema audience during the GermanySlovakia game by monitoring changes in air composition resulting from their exhalations (for methodology, see J. Williams et al. Sci. Rep. 6, 25464; 2016).

In moments of high excitement, exhaled carbon dioxide seems to spike as people's heartbeats and breathing accelerate (see 'Breath chemistry of football fans'). So do emission rates of isoprene, which is released from muscles as fans spring from their seats when a goal is scored. Breath chemistry therefore appears to ride the same emotional roller coaster as the live broadcast.

Christof Stönner, Jonathan Williams Max Planck Institute for Chemistry, Mainz, Germany. jonathan.williams@mpic.de

\section{Journals should drive data reproducibility}

Peer-reviewed journals - as well as researchers and their funders - must take responsibility for improving the reproducibility of published results (see Nature 533, 452-454; 2016).

I suggest that journals should be required to sign a global statement indicating that, to the best of their knowledge, the data that they publish are reproducible. This statement would be collaboratively formulated by the editors-in-chief in accordance with recommendations from the International Committee of Medical Journal Editors and guidelines proposed by the US

\section{BREATH CHEMISTRY OF FOOTBALL FANS}

People exhale bursts of carbon dioxide and isoprene whenever a goal is scored.

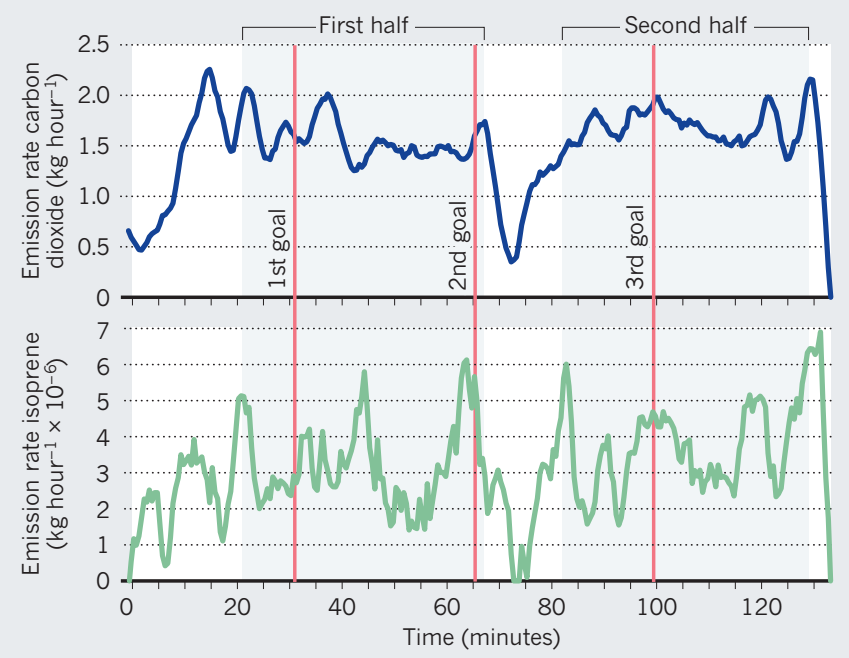

National Institutes of Health, Nature and Science (see Nature 515, 7; 2014 and go.nature. com/29bxphv).

Journals would then publish only papers that are accompanied online by full experimental protocols, raw data and source code, as in the Protocol Exchange repository (www.nature. com/protocolexchange). For manuscripts containing statistical analyses, journals should peer review only those papers that use statistics environments based on source code, enforcing the ban on 'point-and-click' statistical software (see go.nature. com/29pdpcl).

Gregorio Santori University of Genoa, Italy.

gregorio.santori@unige.it

\section{Don't mar legislation with pseudoscience}

We are concerned that some of the European Union's processes for setting safety regulations for chemicals are being influenced by media and pseudoscience scaremongering. Pseudoscience has no place in such decisions, which should be based purely on well-defined and transparent evidence.

For example, endocrine disruptors are being blamed for obesity and type 2 diabetes (J. Legler et al. J. Clin. Endocrinol. Metab. 100, 1278-1288;

2015) despite the absence of supporting evidence for this, and despite food and sugar over-consumption being established as a proven cause. As a consequence, the European Commission's criteria for regulating endocrine-disrupting compounds as a threat to human health are based on correlational, not causal, studies (see go.nature. com/29rjlik).

Conflicts of interest can contribute to the problem, beyond the commercial motivation of industry. Some non-governmental organizations might need to maintain public concerns to boost charitable donations. Decision-makers might prefer to disregard evidence-based data that contradict a precautionary viewpoint. And some scientists put securing research funds above objective appraisal of the evidence.

Acting on hazard identification alone relieves the scaremongering party of the burden of proof, when harm is simply assumed. As a result, regulations can become unnecessarily restrictive. They may even be damaging, for example if an agricultural ban were to be imposed on triazole fungicides because of their endocrine-disrupting potential. The risk to humans at such levels of exposure would be negligible (J.E. Chambers et al. Crit. Rev. Toxicol. 44, 176-210; 2014). It makes no sense to override such evidence with a blanket ban on potentially hazardous chemicals that ignores the public's demonstrable low level of exposure.

Daniel R. Dietrich ${ }^{\star}$ University of Konstanz, Germany. daniel.dietrich@uni-konstanz.de ${ }^{*}$ On behalf of 8 correspondents (see go.nature.com/29kbyqb for full list).

\section{Drug repositioning needs a rethink}

Repurposing drugs to treat illnesses for which they were not originally intended can be faster and cheaper than developing new ones (see Nature 534, 314-316; 2016). I suggest that greater improvements would come from testing different drug combinations, rather than relying only on high-throughput screening of generic or failed drugs.

Disease is often an integration of multiple pathologies (see, for example, J. N. Weinstein et al. Nature 507, 315-322; 2014), so these are potentially treatable with different drug combinations that act in synergy. Such combinations often show better efficacy than single treatments, have fewer side effects and are less likely to result in drug resistance (see Nature 492, 118-122; 2012).

For commercial reasons, pharmaceutical firms tend to dismiss reposition testing of drugs that are off patent. I therefore suggest that governments step in to fund the repurposing of established drugs to broaden the search.

Xianting Ding Shanghai Jiao Tong University, Shanghai, China. dingxianting@sjtu.edu.cn 